\title{
Dirichlet boundary value problem for differential equations involving dry friction
}

\author{
Jan Andres ${ }^{*}$ and Hana Machů
}

${ }^{*}$ Correspondence: jan.andres@upol.cz Department of Mathematical Analysis and Applications of Mathematics, Faculty of Science, Palacký University, 17. listopadu 12, Olomouc, 771 46, Czech Republic

\begin{abstract}
Sufficient conditions in terms of growth restrictions are given for the solvability of the Dirichlet boundary value problem to forced nonlinear differential equations involving the combination of viscous and dry frictions. Explicit estimates of solutions and their derivatives allow us to restrict ourselves to a sufficiently large neighbourhood of the origin, when formulating these effective conditions. In this way, the behaviour of nonlinearities outside of this neighbourhood can be quite arbitrary. In order to get optimal solvability criteria, the problems with one-term and complete linear differential operators will be treated separately by means of various Green's functions. The obtained results are compared with some of their analogies of the other authors.
\end{abstract}

MSC: 34A60; 34B15; 34B16; 34B27; 47H04

Keywords: Dirichlet problem; dry friction; Filippov solution; Green's functions; growth restrictions; Kakutani-Ky Fan fixed point theorem; solution estimates

\section{Introduction}

The Dirichlet problem is one of the mostly studied boundary value problems for differential equations. For ordinary differential equations, the first results due to Hamel, Hammerstein and Lichtenstein were obtained by variational methods (see [1], where also more recent results established in this way are systematically described). After publishing the Schauder fixed point theorem in 1930, topological methods started to be alternatively applied also for vector equations (see e.g. [2-13]). It is well known from the 1930s that, according to the Scorza Dragoni theorem for vector second-order ordinary differential equations with bounded continuous right-hand sides (r.h.s.), the Dirichlet problem is always solvable (see $[12,13])$. Thus, for instance as a very particular case, the Dirichlet problem for a forced mathematical pendulum equation, i.e.

$$
x^{\prime \prime}(t)+b \sin x(t)=p(t), \quad x(0)=x_{0}, \quad x(T)=x_{T},
$$

where $b, x_{0}, x_{T}$ and $T>0$ are real constants, admits a solution, for any $b$ and $p \in$ $C([0, T], \mathbb{R})$. In fact, $p:[0, T] \rightarrow \mathbb{R}$ can be only Lebesgue integrable, i.e. measurable and $\int_{0}^{T}|p(t)| \mathrm{d} t<\infty$, and, for the same goal, the pendulum equation can involve the viscous damping term, i.e.

$$
x^{\prime \prime}(t)+a x^{\prime}(t)+b \sin x(t)=p(t), \quad a \in \mathbb{R}
$$

(see e.g. [6]).

(c) 2015 Andres and Machů. This article is distributed under the terms of the Creative Commons Attribution 4.0 International License (http://creativecommons.org/licenses/by/4.0/), which permits unrestricted use, distribution, and reproduction in any medium, provided you give appropriate credit to the original author(s) and the source, provide a link to the Creative Commons license, and indicate if changes were made. 
On the other hand, the Dirichlet problem for a forced linear oscillator with a viscous friction, i.e.

$$
x^{\prime \prime}(t)+a x^{\prime}(t)+b x(t)=p(t), \quad x(0)=x_{0}, \quad x(T)=x_{T},
$$

has a unique solution, provided again $\int_{0}^{T}|p(t)| \mathrm{d} t<\infty$, but still the homogeneous problem, namely

$$
x^{\prime \prime}(t)+a x^{\prime}(t)+b x(t)=0, \quad x(0)=0, \quad x(T)=0,
$$

should have only a trivial solution which is not always the case; otherwise, the problem is in resonance.

In the presence of a dry friction, the notion of a Carathéodory solution, i.e. the one with an absolutely continuous derivative, is insufficient. The appropriate notion is a Filippov solution which is a Carathéodory solution, but of a differential inclusion with a Filippov regularized right-hand side (see e.g. $[14,15]$ ). For the history and phenomenology of dry friction problems in general, see e.g. [16-20].

For the combination of viscous and dry frictions, it means to consider the problem

$$
x^{\prime \prime}(t)+a x^{\prime}(t)+b \sin x(t)+c \operatorname{sgn} x^{\prime}(t)=p(t), \quad x(0)=x_{0}, \quad x(T)=x_{T},
$$

when dealing with a Filippov solution of a forced pendulum equation, resp. the problem

$$
x^{\prime \prime}(t)+a x^{\prime}(t)+b \sin x(t) \in p(t)-c \operatorname{Sgn} x^{\prime}(t), \quad x(0)=x_{0}, \quad x(T)=x_{T},
$$

where

$$
\operatorname{Sgn} z= \begin{cases}-1, & \text { for } z \in(-\infty, 0) \\ {[-1,1],} & \text { for } z=0 \\ 1, & \text { for } z \in(0, \infty)\end{cases}
$$

when dealing with a Carathéodory solution of a forced pendulum inclusion.

In the case of a forced 'linear'a oscillator, the related problems read as follows:

$$
x^{\prime \prime}(t)+a x^{\prime}(t)+b x(t)+c \operatorname{sgn} x^{\prime}(t)=p(t), \quad x(0)=x_{0}, \quad x(T)=x_{T},
$$

resp.

$$
x^{\prime \prime}(t)+a x^{\prime}(t)+b x(t) \in p(t)-c \operatorname{Sgn} x^{\prime}(t), \quad x(0)=x_{0}, \quad x(T)=x_{T} .
$$

Applying the Kakutani-Ky Fan fixed point theorem (see e.g. Theorem II.8.4. on pp.168169 in [21]), Lasota and Opial (see Theorem 3 in [22]) formulated in 1965 a theorem for a first-order vector problem. This theorem solves problem (2) resp. (1), for any $a$, $b, c$, provided only $\int_{0}^{T}|p(t)| \mathrm{d} t<\infty$, as well as problem (5) resp. (4), provided again $\int_{0}^{T}|p(t)| \mathrm{d} t<\infty$, and additionally requiring either $|b| T$ to be sufficiently small or a trivial solvability of a homogeneous problem

$$
x^{\prime \prime}(t)+a x^{\prime}(t)+b x(t)=0, \quad x(0)=x_{0}, \quad x(T)=x_{T} .
$$


On the other hand, the estimates of solutions and derivatives are not indicated explicitly in [22].

There are also further related results concerning problems (2) and (5) obtained mainly by means of degree arguments (see e.g. [23-34]), but none of them allows us to get such simple criteria as those in [22].

For instance, according to Theorem 6.1 in [25] as well as Theorem 2 in [33], where the combination of sign and growth restriction was employed, both problems (2) and (5) are solvable only in the lack of a viscous friction, i.e. only for $a=0$, provided $\int_{0}^{T}|p(t)| \mathrm{d} t<$ $\infty$ for (2), and $\int_{0}^{T}|p(t)| \mathrm{d} t<\infty$ together with $b \leq 0$ for (5). Moreover, the estimates of solutions are again not indicated explicitly in $[25,33]$.

Similarly, in Theorem 4.1 in [26], Theorem 4.1 in [29] and Theorem 3.1 in [30], the viscous friction term $a x^{\prime}$ cannot be involved in (2) and (5) but, under more restrictive assumptions than those in $[25,33]$, the solution estimates are available there.

Using the Hartman-type conditions (called along the lines of the classical paper [8]), as in Corollary 4.1 in [23], the related sufficient conditions for the solvability of (2) are again more restrictive than those in Theorem 3 in [22], namely $b<0, p$ is continuous on $[0, T]$, and

$$
\frac{P+|c|+k_{3}}{-b} \leq \sin \left(\frac{\pi}{2}-k_{2}\right), \quad k_{2} \leq \frac{\pi}{2},
$$

where

$$
k_{2}:=\max \left\{\left|x_{0}\right|,\left|x_{T}\right|\right\}, \quad k_{3}:=\frac{\left|x_{T}-x_{0}\right|}{T}, \quad P:=\max _{t \in[0, T]}|p(t)|,
$$

but $a$ can be this time different from zero, i.e. $a \neq 0$.

Those for the solvability of (5) hold, according to Corollary 4.1 in [23], without (6), i.e. with $b<0$ and $p$ is continuous on $[0, T](\Rightarrow|p(t)| \leq P, t \in[0, T])$, only.

In the absence of a viscous friction, i.e. when $a=0$, the forcing term $p$ can be, according to Corollary 4.3 in [23] and Theorem 3 in [30], Lebesgue measurable and essentially bounded, for both problems (2) and (5). For $b=0$, however, it should be $c=0, p(t) \equiv 0$ and $k_{3}=0$ or $a=0$ in order Corollaries 4.1 and 4.3 in [23] and Theorem 3 in [30] to be applied to (2) and (5) which reduces to a trivial solvability of (2) and (5).

On the other hand, under the above assumptions with $b<0$, the solution $x(\cdot)$ estimates for (2) and (5) can be this time expressed explicitly as

$$
|x(t)| \leq \arcsin \left(\frac{P+|c|+k_{3}}{-b}\right)-k_{2} \leq \frac{\pi}{2}-k_{2}
$$

and

$$
|x(t)| \leq \frac{P+|c|+k_{3}}{-b}+k_{2},
$$

respectively.

If further possibly discontinuous nonlinearities or multivalued maps are implemented into the right-hand sides of given differential equations or inclusions whose growth has, for instance, a superlinear character sufficiently far from the origin, then Theorem 3 in [22] 
does not any longer apply. Having, however, to our disposal explicit estimates of solutions like (7) or (8) and their derivatives, we can formulate criteria such that the implemented new terms can behave in an arbitrary way outside of the domains characterized by these estimates. In this way, all the results under our consideration can be naturally extended.

This will be therefore our main aim of the present paper. Of course, for obtaining the explicit estimates of solutions and their derivatives, we should study, unlike in [22], exclusively the second-order Dirichlet boundary value problems. For the sake of brevity we will prove only one main theorem for the scalar problem. Nevertheless, since all the related proofs of all the cases under our consideration are quite analogous and differ just by the technical details, we can also present the related solvability criteria and some solutions estimates.

On the other hand, we will discuss in detail the advantages and disadvantages of the usage of one-term vs. complete linear differential operator for the associated Green's functions.

Hence, our paper will be organized as follows. After the preliminaries, the scalar multivalued Dirichlet problem of the form

$$
\left.\begin{array}{l}
x^{\prime \prime}(t)+a x^{\prime}(t)+b x(t) \in P(t)+F_{1}(x(t))+F_{2}\left(x^{\prime}(t)\right)-c \operatorname{Sgn} x^{\prime}(t) \\
x(0)=x_{0}, \quad x(T)=x_{T}
\end{array}\right\}
$$

will be considered. We will distinguish between the one-term linear differential operator $L x:=x^{\prime \prime}$ (then the remaining terms $a x^{\prime}+b x$ will be considered in Section 3 as a part of a multivalued perturbation of the r.h.s. of the inclusion in (9)) and the complete linear differential operator $L x:=x^{\prime \prime}+a x^{\prime}+b x$ in Section 4, where particular vector analogies of some obtained results are also indicated.

\section{Preliminaries}

Let $X, Y$ be two metric spaces. We say that $\varphi$ is a multivalued mapping from $X$ to $Y$ (written $\varphi: X \multimap Y$ ) if, for every $x \in X$, a nonempty closed subset $\varphi(x)$ of $Y$ is given. We can associate with $\varphi$ its graph $\Gamma_{\varphi}$, the subset of $X \times Y$, defined by

$$
\Gamma_{\varphi}:=\{(x, y) \in X \times Y \mid y \in \varphi(x)\}
$$

A multivalued mapping $\varphi: X \multimap Y$ is called upper semicontinuous (u.s.c.) if, for each open $U \subset Y$, the set $\{x \in X \mid \varphi(x) \subset U\}$ is open in $X$.

Lemma 1 (cf., e.g., Proposition I.3.15 in [14]) If $\varphi: X \multimap Y$ is u.s.c., then the graph $\Gamma_{\varphi}$ is a closed subset of $X \times Y$.

Lemma 2 (cf., e.g., Proposition I.3.16 in [14]) Assume that $\varphi: X \multimap Y$ is a multivalued mapping such that $\varphi(X) \subset K$, where $K \subset Y$ is a compact set, and the graph $\Gamma_{\varphi}$ of $\varphi$ is closed. Then $\varphi$ is u.s.c.

Let $Y$ be a separable metric space and $(\Omega, \mathcal{U}, \mu)$ be a measurable space, i.e. a set $\Omega$ equipped with a $\sigma$-algebra $\mathcal{U}$ of subsets and a countably additive measure $\mu$ on $\mathcal{U}$. For our needs here, $\Omega$ will be a bounded domain in $\mathbb{R}^{k}$, equipped with the Lebesgue measure. 
A multivalued mapping $\varphi: \Omega \multimap Y$ is called measurable if $\{\omega \in \Omega \mid \varphi(\omega) \subset V\} \in \mathcal{U}$, for each open set $V \subset Y$.

A multivalued mapping $F: J \times \mathbb{R}^{n} \multimap \mathbb{R}^{m}$ with compact and convex values, where $J \subset \mathbb{R}$ is a compact interval, is an upper-Carathéodory (shortly, u-Carathéodory) mapping if it satisfies

(i) $t \multimap F(t, x)$ is measurable, for every $x \in \mathbb{R}^{n}$,

(ii) $x \multimap F(t, x)$ is u.s.c., for almost all (a.a.) $t \in J$,

(iii) $|y| \leq r(t)(1+|x|)$, for every $(t, x) \in J \times \mathbb{R}^{n}$, and every $y \in F(t, x)$, where $r: J \rightarrow[0, \infty)$ is a Lebesgue integrable function.

Let $F: X \multimap Y$ be a multivalued mapping and $f: X \rightarrow Y$ be a single-valued mapping. We say that $f$ is a selection of $F$ (written $f \subset F$ ) if $f(x) \in F(x)$, for every $x \in X$.

We will employ the following definitions and statements.

Proposition 1 (Castaing representation, $c f$. Theorem III.7 in [35]) Let $X$ be a separable metric space, $(\Omega, \mathcal{U}, \mu)$ a measurable space, and $\varphi$ a measurable multivalued mapping from $\Omega$ to complete subsets of $X$. Then there exists a sequence $\left\{f_{n}\right\}$ of measurable selections of $\varphi$ such that, for every $x$,

$$
\varphi(x)=\overline{\left\{f_{n}(x) \mid n \in \mathbb{N}\right\}}=\overline{\bigcup_{n \in \mathbb{N}} f_{n}(x)},
$$

where the bar denotes the closure in $X$.

Lemma 3 (cf., e.g., Lemma 7.1 in [36]) Let $F: J \times \mathbb{R}^{n} \multimap \mathbb{R}^{n}$ be a u-Carathéodory multivalued mapping. Then the composition $F(t, q(t))$ admits, for every $q \in C\left(J, \mathbb{R}^{n}\right)$, a single-valued measurable selection.

If $X \subset Y$ and $\varphi: X \multimap Y$, then a point $x \in X$ is called a fixed point of $\varphi$ if $x \in \varphi(x)$. We set $\operatorname{Fix}(\varphi):=\{x \in X \mid x \in \varphi(x)\}$.

Definition 1 Let $X$ and $Y$ be subsets of normed linear spaces and $\varphi: X \multimap Y$ be a multivalued mapping. If $Y$ is convex, then $\varphi$ is called a Kakutani map, provided $\varphi$ is u.s.c. with (nonempty) compact, convex values.

Let $X$ and $Y$ be metric spaces. A multivalued mapping $\varphi: X \multimap Y$ is called compact if its image $\varphi(X)=\bigcup\{\varphi(x) \mid x \in X\}$ is contained in a compact subset of $Y$.

The following statement, which we state in the form of proposition, is usually called the Kakutani-Ky Fan-type fixed point theorem.

Proposition 2 (cf., e.g., Theorem II.8.4 in [21]) Let C be a convex (not necessarily closed) subset of a normed linear space, and let $\varphi: C \multimap C$ be a compact Kakutani map. Then $\varphi$ has a fixed point.

Definition 2 Let $\varphi: J \times \mathbb{R}^{n} \multimap \mathbb{R}^{n}$ be a convex-valued, bounded and measurable mapping. Then the mapping

$$
\Phi(t, x)=\bigcap_{\delta>0} \bigcap_{\mu(N)=0} \overline{\operatorname{conv}} \varphi(B((t, x), \delta) \backslash N)
$$


is called the Filippov-like regularization of $\varphi$, where $\mu$ denotes Lebesgue measure on $\mathbb{R}^{n}$, $N \subset \mathbb{R}^{n}$, and conv denotes the closed convex hull of a set. $B((t, x), \delta)$ is an open ball of radius $\delta>0$, centered at a point $(t, x)$.

Observe that $\Phi$ is a bounded u.s.c. mapping with (nonempty) compact and convex values.

Let $P: J \multimap \mathbb{R}^{n}$ be a multivalued mapping having a nonempty set of Lebesgue integrable selections. The Aumann integral of $P$ is defined as follows ( $c f$. [37]):

$$
\int_{J} P(t) \mathrm{d} t:=\left\{\int_{J} p(t) \mathrm{d} t \mid p \subset P \text { is a Lebesgue integrable selection of } P\right\} .
$$

Now, we shall consider the vector boundary value problem (BVP) in $\mathbb{R}^{n}$ :

$$
L u(t)=f(t), \quad a \leq t \leq b, a, b \in \mathbb{R}
$$

where $L$ is a linear ordinary differential operator of order 2 and $f:[a, b] \rightarrow \mathbb{R}^{n}$ is a Lebesgue measurable function in $a \leq t \leq b$, with homogeneous boundary conditions

$$
u(a)=0, \quad u(b)=0 .
$$

Proposition 3 (cf., e.g., Theorem 3.2.1 in [38]) If the homogeneous BVP associated with (10), (11), i.e. the one with $f(t) \equiv 0$, has only a trivial solution, then the BVP (10), (11) has exactly one solution which is given by

$$
u(t)=\int_{a}^{b} G(t, s) f(s) \mathrm{d} s
$$

where $G:[a, b] \times[a, b] \rightarrow \mathbb{R}$ is the Green's function related to the homogeneous BVP associated with (10), (11).

It will also be convenient to recall the following lemmas.

Lemma 4 (cf., e.g., Theorem 0.3.4 in [39]) Assume that the sequence of absolutely continuous functions $x_{k}: J \rightarrow \mathbb{R}^{n}$, where $J$ is a compact interval, satisfies the following conditions:

- the set $\left\{x_{k}(t) \mid k \in \mathbb{N}\right\}$ is bounded $\forall t \in J$,

- there is a Lebesgue integrable function $\alpha: J \rightarrow \mathbb{R}$ such that

$$
\left|x_{k}^{\prime}(t)\right| \leq \alpha(t), \quad \text { for almost all } t \in J, \forall k \in \mathbb{N} \text {. }
$$

Then there exists a subsequence $\left\{x_{k}\right\}$ (denoted just the same) convergent to an absolutely continuous function $x: J \rightarrow \mathbb{R}^{n}$ in the following sense:

(i) $\left\{x_{k}\right\}$ uniformly converges to $x$,

(ii) $\left\{x_{k}^{\prime}\right\}$ weakly converges in $L^{1}\left(J, \mathbb{R}^{n}\right)$ to $x^{\prime}$.

Lemma 5 (cf. [40], p.88) Let $J \subset \mathbb{R}$ be a compact interval and $F: J \times \mathbb{R}^{n} \multimap \mathbb{R}^{n}$ be a $u$-Carathéodory mapping. Let $N_{F}: C\left(J, \mathbb{R}^{n}\right) \multimap L^{1}\left(J, \mathbb{R}^{n}\right)$ be the Nemytskil operator defined 
as follows:

$$
N_{F}(x):=\left\{f \in L^{1}\left(J, \mathbb{R}^{n}\right) \mid f(t) \in F(t, x(t)), \text { for almost all } t \in J\right\},
$$

for every $x \in C\left(J, \mathbb{R}^{n}\right)$. Then, if the sequences $\left\{x_{i}\right\} \subset C\left(J, \mathbb{R}^{n}\right)$ and $\left\{f_{i}\right\} \subset L^{1}\left(J, \mathbb{R}^{n}\right), f_{i} \in N_{F}\left(x_{i}\right)$, $i \in \mathbb{N}$, are such that $x_{i} \rightarrow x$ in $C\left(J, \mathbb{R}^{n}\right)$ and $f_{i} \rightarrow f$ weakly in $L^{1}\left(J, \mathbb{R}^{n}\right)$, then $f \in N_{F}(x)$.

\section{Main theorem}

Let us consider at first the scalar Dirichlet problem for differential equations involving a dry friction (4), where $a, b, c, x_{0}, x_{T}$ and $T>0$ are real constants and $p: J \rightarrow \mathbb{R}, J=[0, T]$, is a Lebesgue integrable function.

Since the function $\operatorname{sgn}(\cdot)$ is discontinuous in the spatial variable, problem (4) need not have a Carathéodory solution, i.e. a function $x: J \rightarrow \mathbb{R}$ with an absolutely continuous derivative, satisfying (4), for almost all $t \in J$. Therefore, we need another notion of an appropriate solution, namely the one in the sense of Filippov. For this goal, we use the concept of the Filippov-like regularization (see [15]) of spatially discontinuous maps. More precisely, applying Definition 2 to the right-hand side involving spatial discontinuities, we can speak about a solution in the sense of Filippov of the original problem, provided it is a Carathéodory solution of a multivalued problem with a Filippov-like regularized righthand side.

In our situation, the discontinuous function to be regularized is the function signum. On the basis of the Filippov-like regularization of $\operatorname{sgn}(\cdot)$, we obtain the multivalued mapping Signum defined in (3), i.e. $\operatorname{Sgn}(\cdot)$.

One can readily check that the Signum mapping is u.s.c. with compact and convex values. Hence, after the described Filippov-like regularization, problem (4) with a discontinuous function $\operatorname{sgn}(\cdot)$ becomes multivalued, i.e. (5).

By a Filippov solution of (4), we understand a function $x(\cdot): J \rightarrow \mathbb{R}$ with absolutely continuous derivative, satisfying problem (5), almost everywhere on $J$.

For our needs, it will be convenient to consider still the problem involving more multivalued terms, namely (9), where $F_{1}: \mathbb{R} \multimap \mathbb{R}, F_{2}: \mathbb{R} \multimap \mathbb{R}$ are u.s.c. maps with compact, convex values and $P: J \multimap \mathbb{R}$ is an Aumann integrable mapping, $J=[0, T]$.

Making the change of variables $y(t)=x(t)-v(t)$, where $v(t)=\frac{x_{T}-x_{0}}{T} t+x_{0}$, we can immediately see that $x(t)$ is a Carathéodory solution of (9) if and only if $y(t)$ satisfies

$$
\left.\begin{array}{l}
y^{\prime \prime}(t)+a\left(y^{\prime}(t)+v^{\prime}(t)\right)+b(y(t)+v(t)) \\
\quad \in P(t)+F_{1}(y(t)+v(t))+F_{2}\left(y^{\prime}(t)+v^{\prime}(t)\right)-c \operatorname{Sgn}\left(y^{\prime}(t)+v^{\prime}(t)\right), \quad \text { for a.a. } t \in J, \\
y(0)=0, \quad y(T)=0 .
\end{array}\right\}
$$

One can also easily check that $|v(t)| \leq k_{2}:=\max \left\{\left|x_{0}\right|,\left|x_{T}\right|\right\}$, for all $t \in J$, and $\left|v^{\prime}(t)\right|=k_{3}:=$ $\frac{\left|x_{T}-x_{0}\right|}{T}$, for all $t \in J$.

Our aim at this moment is to prove the existence and localization theorems for problem (9). Since the desired sufficient conditions will tendentiously take the form of growth restrictions, let us start with the problem involving the truncated maps of $F_{1}, F_{2}$, i.e.

$$
\begin{aligned}
& y^{\prime \prime}(t)+a y^{\prime}(t)+b y(t) \\
& \quad \in P(t)-a v^{\prime}(t)-b v(t)+F_{1}^{*}(y(t)+v(t)) \\
& \quad+F_{2}^{*}\left(y^{\prime}(t)+v^{\prime}(t)\right)-c \operatorname{Sgn}\left(y^{\prime}(t)+v^{\prime}(t)\right), \quad \text { for a.a. } t \in J, \\
& y(0)=0, \quad y(T)=0,
\end{aligned}
$$


where

$$
\begin{aligned}
& F_{1}^{*}(z):= \begin{cases}F_{1}(z), & \text { for }|z| \leq D, \\
F_{1}(D \operatorname{sgn}(z)), & \text { for }|z| \geq D,\end{cases} \\
& F_{2}^{*}(z):= \begin{cases}F_{2}(z), & \text { for }|z| \leq D, \\
F_{2}(D \operatorname{sgn}(z)), & \text { for }|z| \geq D .\end{cases}
\end{aligned}
$$

Obviously, $\left.F_{1}\right|_{\bar{B}_{D}}: \bar{B}_{D} \multimap \mathbb{R}$ and $\left.F_{2}\right|_{\bar{B}_{D}}: \bar{B}_{D} \multimap \mathbb{R}$ are u.s.c. maps with compact and convex values, where $\bar{B}_{D}:=\{z \in \mathbb{R}|| z \mid \leq D\}$ is the closed ball, constant $D$ is such that $D=D_{0}+k_{2}+$ $k_{3}$, where $D_{0}>0$ is a suitable constant which will be specified later, and $k_{2}, k_{3}$ are defined above. Thus, the same is true for $F_{1}^{*}, F_{2}^{*}: \mathbb{R} \multimap \mathbb{R}$ defined as above.

Observe that, in this way, $F_{1}^{*}(z+v(t))=F_{1}(z+v(t))$, for $|z| \leq D_{0}$, and $F_{2}^{*}\left(z+v^{\prime}(t)\right)=F_{2}(z+$ $\left.v^{\prime}(t)\right)$, for $|z| \leq D_{0}$.

Hence, let us find sufficient conditions for the solvability of (13). We distinguish two cases in order to separate formally a linear differential operator and a multivalued perturbation.

In this section, we will deal with the problem formally written in the following way:

$$
\left.\begin{array}{l}
y^{\prime \prime}(t) \in F\left(t, y(t), y^{\prime}(t)\right), \quad \text { for a.a. } t \in J, \\
y(0)=0, \quad y(T)=0,
\end{array}\right\}
$$

where $F\left(t, y(t), y^{\prime}(t)\right):=P(t)+F_{1}^{*}(y(t)+v(t))+F_{2}^{*}\left(y^{\prime}(t)+v^{\prime}(t)\right)-a\left(y^{\prime}(t)+v^{\prime}(t)\right)-b(y(t)+v(t))-$ $c \operatorname{Sgn}\left(y^{\prime}(t)+v^{\prime}(t)\right)$, i.e. $F: J \times \mathbb{R} \times \mathbb{R} \multimap \mathbb{R}$ is a u-Carathéodory multivalued mapping and $L y(t):=y^{\prime \prime}(t)$.

For a solvability of the multivalued nonlinear problem (14), we use the Schauder linearization device. Thus, we parametrize the right-hand side (r.h.s.) $F$ in order to have a one-parameter family of linear problems. Let

$$
Q:=\left\{u \in C^{1}(J, \mathbb{R}),\|u\|_{C^{1}} \leq D_{0}\right\}
$$

be the set of candidate solutions, where $\|u\|_{C^{1}}:=\sup \left\{|u(t)|+\left|u^{\prime}(t)\right|, t \in J\right\}$ and $D_{0}>0$ is a suitable constant.

Then, for each $q \in Q$, we get a fully linearized problem

$$
\left.\begin{array}{l}
y^{\prime \prime}(t) \in F_{q}(t), \quad \text { for a.a. } t \in J, \\
y(0)=0, \quad y(T)=0,
\end{array}\right\}
$$

where $F_{q}(t)=P(t)+F_{1}^{*}(q(t)+v(t))+F_{2}^{*}\left(q^{\prime}(t)+v^{\prime}(t)\right)-a q^{\prime}(t)-a v^{\prime}(t)-b q(t)-b v(t)-$ $c \operatorname{Sgn}\left(q^{\prime}(t)+v^{\prime}(t)\right)$. Obviously, $F_{q}: J \multimap \mathbb{R}$ is, for every $q \in Q$, an Aumann integrable function of $t$.

From Lemma 3, the existence follows of at least one measurable selection $f_{q} \subset F_{q}$ of the multivalued composition $F_{q}(t)$. Thus, we can consider the single-valued linear Dirichlet problem

$$
\left.\begin{array}{l}
y^{\prime \prime}(t)=f_{q}(t), \quad \text { for a.a. } t \in J, \\
y(0)=0, \quad y(T)=0 .
\end{array}\right\}
$$


The homogeneous problem associated with the problem (16), i.e.

$$
\left.\begin{array}{l}
y^{\prime \prime}(t)=0, \quad \text { for a.a. } t \in J, \\
y(0)=0, \quad y(T)=0,
\end{array}\right\}
$$

has only a trivial solution. Hence, it follows from the Fredholm alternative (see Proposition 3) that problem (16) has a unique Carathéodory solution $y(\cdot)$, which takes the form

$$
y(t)=\int_{0}^{T} G_{1}(t, s) f_{q}(s) \mathrm{d} s,
$$

where $G_{1}$ is the Green's function of problem (17), i.e.

$$
G_{1}(t, s)= \begin{cases}\frac{t(s-T)}{T}, & \text { for all } 0 \leq t \leq s \leq T, \\ \frac{s(t-T)}{T}, & \text { for all } 0 \leq s \leq t \leq T .\end{cases}
$$

One can easily check that $\left|G_{1}(t, s)\right| \leq \frac{T}{4}$, for all $t, s \in[0, T]$.

In fact, since the multivalued composition $F_{q}(t)$ is, for every $q \in Q$, obviously measurable, according to Proposition 1, we can even write

$$
y(t) \in \int_{0}^{T} G_{1}(t, s) \overline{\bigcup_{n \in \mathbb{N}} f_{n, q}(s)} \mathrm{d} s,
$$

where $\left\{f_{n, q}(t) \subset F_{q}(t)\right\}_{n \in \mathbb{N}}$ is a sequence of measurable selections of $F_{q}$ and the integral is understood in the sense of Aumann.

Observe that because of

$$
\frac{\partial G_{1}}{\partial t}(t, s)= \begin{cases}\frac{(s-T)}{T}, & \text { for all } 0 \leq t \leq s \leq T, \\ \frac{s}{T}, & \text { for all } 0 \leq s \leq t \leq T,\end{cases}
$$

we also have

$$
y^{\prime}(t) \in \int_{0}^{T} \frac{\partial G_{1}}{\partial t}(t, s) \overline{\bigcup_{n \in \mathbb{N}} f_{n, q}(s)} \mathrm{d} s
$$

where the integral is again understood in the sense of Aumann. Moreover, $\left|\frac{\partial G_{1}}{\partial t}(t, s)\right| \leq 1$, for all $t, s \in[0, T]$.

Hence, denoting by $\varphi: Q \multimap C^{1}(J, \mathbb{R})$ the solution operator of (15), where

$$
\varphi:=\int_{0}^{T} G_{1}(t, s) F_{q}(s) \mathrm{d} s=\int_{0}^{T} G_{1}(t, s) \overline{\bigcup_{n \in \mathbb{N}} f_{n, q}(s)} \mathrm{d} s
$$

instead of the existence of the solution of differential problem (15), we can equivalently investigate the existence of a fixed point of the multivalued operator $\varphi$. For this purpose, we will apply the Kakutani-Ky Fan-type fixed point theorem (Proposition 2). In this way, we will prove the following theorem. 
Theorem 1 Let $a, b, c, x_{0}, x_{T}$ and $T>0$ be real constants such that

$$
\frac{4}{T(T+4)}>\max \{|a|,|b|\} \text {. }
$$

Assume that $P: J \multimap \mathbb{R}$ is an Aumann integrable multivalued mapping and $\left.F_{1}\right|_{\bar{B}_{D}}: \bar{B}_{D} \multimap \mathbb{R}$, $\left.F_{2}\right|_{\bar{B}_{D}}: \bar{B}_{D} \multimap \mathbb{R}$ are u.s.c. multivalued mappings with convex and compact values, where $D=D_{0}+k_{2}+k_{3}, D_{0}>0$ is still supposed to be a suitable constant such that

$$
D_{0} \geq \Delta_{1}\left(D_{0}\right),
$$

where

$$
\Delta_{1}\left(D_{0}\right):=\frac{\left[\mathcal{P}+T\left(M_{1}\left(D_{0}\right)+M_{2}\left(D_{0}\right)+|a| k_{3}+|b| k_{2}+|c|\right)\right](T+4)}{4-k_{1} T(T+4)}
$$

and

$$
\begin{aligned}
& M_{1}\left(D_{0}\right):=\max _{|z| \leq D_{0}+k_{2}+k_{3}}\left|F_{1}(z)\right|, \quad M_{2}\left(D_{0}\right):=\max _{|z| \leq D_{0}+k_{2}+k_{3}}\left|F_{2}(z)\right|, \\
& k_{1}:=\max \{|a|,|b|\}, \quad k_{2}:=\max \left\{\left|x_{0}\right|,\left|x_{T}\right|\right\}, \quad k_{3}:=\frac{\left|x_{T}-x_{0}\right|}{T}, \\
& \mathcal{P}:=\sup _{p \subset P}\left\{\int_{0}^{T}|p(t)| \mathrm{d} t \mid p \subset P \text { is a Lebesgue integrable selection of } P\right\} .
\end{aligned}
$$

Then problem (9) admits a solution $x(\cdot)$ such that

$$
\max _{t \in J}\left\{|x(t)|+\left|x^{\prime}(t)\right|\right\} \leq D
$$

Proof In order to check all the assumptions of Proposition 2, we will proceed in four steps.

(i) Since problem (16) is uniquely solvable, the set $\varphi(Q)$ is nonempty.

(ii) Let us prove that the set $\varphi(Q)$, i.e. the set of solutions of (15), is relatively compact. According to the well known Arzelá-Ascoli lemma, the set of solutions is relatively compact in $C^{1}(J, \mathbb{R})$ if and only if it is uniformly bounded and equi-continuous, both in the $C^{1}$-norm.

(a) Let us show that the set of solutions of (15) is uniformly bounded in $C^{1}(J, \mathbb{R})$. Let $u(\cdot)$ be a solution of (15) and $f_{q} \subset F_{q}$ be a measurable selection of $F_{q}$. We could see that such a measurable selection exists and that $u(\cdot)$ takes the form

$$
u(t)=\int_{0}^{T} G_{1}(t, s) f_{q}(s) \mathrm{d} s
$$

It is obvious that

$$
\begin{aligned}
& \max _{|z| \leq D_{0}}\left|F_{1}(z+v(t))\right|=\max _{|z| \leq D_{0}}\left|F_{1}^{*}(z+v(t))\right| \leq M_{1}\left(D_{0}\right), \\
& \max _{|z| \leq D_{0}}\left|F_{2}\left(z+v^{\prime}(t)\right)\right|=\max _{|z| \leq D_{0}}\left|F_{2}^{*}\left(z+v^{\prime}(t)\right)\right| \leq M_{2}\left(D_{0}\right),
\end{aligned}
$$

where $v(t)=\frac{x_{T}-x_{0}}{T} t+x_{0}$ and $v^{\prime}(t)=\frac{x_{T}-x_{0}}{T}$. 
By means of Lemma 3 and in view of (18), (19), for any $t \in J$, we obtain the following estimate:

$$
\begin{aligned}
|u(t)|+\left|u^{\prime}(t)\right|= & \left|\int_{0}^{T} G_{1}(t, s) f_{q}(s) \mathrm{d} s\right|+\left|\int_{0}^{T} \frac{\partial G_{1}}{\partial t}(t, s) f_{q}(s) \mathrm{d} s\right| \\
\leq & \left(\frac{T}{4}+1\right) \int_{0}^{T}\left|f_{q}(s)\right| \mathrm{d} s \\
\leq & \left(\frac{T}{4}+1\right) \int_{0}^{T}|p(s)| \\
& +\left|f_{1}^{*}(q(s)+v(s))\right|+\left|f_{2}^{*}\left(q^{\prime}(s)+v^{\prime}(s)\right)\right|+\left|a\left(q^{\prime}(s)+v^{\prime}(s)\right)\right| \\
& +|b(q(s)+v(s))|+\left|c \operatorname{Sgn}_{\mathrm{Sel}}\left(q^{\prime}(s)+v^{\prime}(s)\right)\right| \mathrm{d} s \\
\leq & \left(\frac{T}{4}+1\right)\left[\mathcal{P}+T\left(M_{1}\left(D_{0}\right)+M_{2}\left(D_{0}\right)+|a| k_{3}+|b| k_{2}+|c|\right)\right. \\
& \left.+k_{1} \int_{0}^{T}|q(s)|+\left|q^{\prime}(s)\right| \mathrm{d} s\right], \\
\leq & \left(\frac{T}{4}+1\right)\left[\mathcal{P}+T\left(M_{1}\left(D_{0}\right)+M_{2}\left(D_{0}\right)+|a| k_{3}+|b| k_{2}+|c|+k_{1} D_{0}\right)\right]
\end{aligned}
$$

where $f_{1}^{*} \subset F_{1}^{*}, f_{2}^{*} \subset F_{2}^{*}, p \subset P$ and $\mathrm{Sgn}_{\text {Sel }} \subset$ Sgn are the related measurable selections.

Since this estimate holds in the same way for all $q \in Q$, this already means that the solutions $u(\cdot)$ of (15), i.e. the set $\varphi(Q)$, are uniformly bounded in the $C^{1}$-norm.

Moreover, according to (20), (22), if there exists a positive constant $D_{0}$ such that $D_{0} \geq$ $\Delta_{1}\left(D_{0}\right)$, then the set $\varphi(Q)$ satisfies $\varphi(Q) \subset Q$.

(b) Now, let us show that the elements of the set $\varphi(Q)$, i.e. the solutions $u(\cdot)$ of (15), are equi-continuous in the $C^{1}$-norm. For any $t_{1}, t_{2} \in J$ with $t_{1}<t_{2}$, we have by means of Lemma 3 and in view of (19)

$$
\begin{aligned}
\left|u\left(t_{2}\right)-u\left(t_{1}\right)\right| & =\left|\int_{t_{1}}^{t_{2}} u^{\prime}(t) \mathrm{d} t\right|=\left|\int_{t_{1}}^{t_{2}} \int_{0}^{T} \frac{\partial G_{1}}{\partial t}(t, s) f_{q}(s) \mathrm{d} s \mathrm{~d} t\right| \\
& \leq\left|\int_{t_{1}}^{t_{2}} \int_{0}^{T}\right| \frac{\partial G_{1}}{\partial t}(t, s)|| f_{q}(s)|\mathrm{d} s \mathrm{~d} t| \leq\left|\int_{t_{1}}^{t_{2}} \int_{0}^{T}\right| f_{q}(s)|\mathrm{d} s \mathrm{~d} t| \\
& \leq\left(t_{2}-t_{1}\right)\left[\mathcal{P}+\left(M_{1}\left(D_{0}\right)+M_{2}\left(D_{0}\right)+k_{1} D_{0}+|a| k_{3}+|b| k_{2}+|c|\right) T\right] .
\end{aligned}
$$

Furthermore, we have still

$$
\begin{aligned}
\left|u^{\prime}\left(t_{2}\right)-u^{\prime}\left(t_{1}\right)\right|= & \left|\int_{t_{1}}^{t_{2}} u^{\prime \prime}(t) \mathrm{d} t\right|=\left|\int_{t_{1}}^{t_{2}} f_{q}(t) \mathrm{d} t\right| \leq\left|\int_{t_{1}}^{t_{2}}\right| f_{q}(t)|\mathrm{d} t| \\
\leq & \left(t_{2}-t_{1}\right)\left[M_{1}\left(D_{0}\right)+M_{2}\left(D_{0}\right)+k_{1} D_{0}+|a| k_{3}+|b| k_{2}+|c|\right] \\
& +\int_{t_{1}}^{t_{2}}|p(t)| \mathrm{d} t .
\end{aligned}
$$

Therefore, the solutions $u(\cdot)$ of (15) are equi-continuous in the $C^{1}$-norm. Summing up (a) and (b), the elements of the set $\varphi(Q)$ are relatively compact in the $C^{1}$-norm, as claimed. (iii) We will show that the operator $\varphi$ is u.s.c. In view of Lemma 2 , and since $\varphi$ was shown to be compact, it is sufficient to show that the graph $\Gamma_{\varphi}$ is closed. Let $\left\{\left(q_{k}, u_{k}\right)\right\} \subset \Gamma_{\varphi}$ be a 
sequence such that $\left\{\left(q_{k}, q_{k}^{\prime}, u_{k}\right)\right\} \rightarrow\left(q, q^{\prime}, u\right)$, where $q \in Q$. For all $k \in \mathbb{N}$ and a.a. $t \in J$, the sequence $\left\{u_{k}^{\prime}\right\}$ is bounded and $\left|u_{k}^{\prime \prime}(t)\right| \leq|p(t)|+M_{1}\left(D_{0}\right)+M_{2}\left(D_{0}\right)+k_{1} D_{0}+|c|+|a| k_{3}+|b| k_{2}$, for a.a. $t \in J$. The sequence $\left\{w_{k}:=u_{k}^{\prime}\right\}$ satisfies all the assumptions of Lemma 4.

Thus, applying Lemma 4 to the sequence $\left\{w_{k}:=u_{k}^{\prime}\right\}$, we find that there exists a subsequence of $\left\{u_{k}^{\prime}\right\}$, for the sake of simplicity denoted in the same way as the sequence, which converges uniformly to $u^{\prime}$ on $J$ and such that $\left\{u_{k}^{\prime \prime}\right\}$ converges weakly to $u^{\prime \prime}$ in $L^{1}(J, \mathbb{R})$.

If we set $z_{k}:=\left(u_{k}, w_{k}\right)$, then $z_{k}^{\prime}=\left(u_{k}^{\prime}, w_{k}^{\prime}\right)=\left(u_{k}^{\prime}, u_{k}^{\prime \prime}\right) \rightarrow\left(u^{\prime}, u^{\prime \prime}\right)$, weakly in $L^{1}\left(J, \mathbb{R}^{2}\right)$. Let us consider the system

$$
z_{k}^{\prime}(t) \in H\left(t, q_{k}(t), q_{k}^{\prime}(t)\right), \quad \text { for a.a. } t \in J,
$$

where $z_{k}^{\prime}(t)=\left(u_{k}^{\prime}(t), w_{k}^{\prime}(t)\right)$ and $H\left(t, q_{k}(t), q_{k}^{\prime}(t)\right)=\left(w_{k}, F_{q_{k}}(t)\right)$.

Applying Lemma 5 , for $f_{i}:=z_{k}^{\prime}, f:=\left(u^{\prime}, u^{\prime \prime}\right), x_{i}:=\left(q_{k}, q_{k}^{\prime}\right)$, it follows that

$$
\left(u^{\prime}(t), u^{\prime \prime}(t)\right) \in H\left(t, q(t), q^{\prime}(t)\right), \quad \text { for a.a. } t \in J,
$$

i.e. $u^{\prime \prime}(t) \in F_{q}(t)$, for a.a. $t \in J$.

The set $\varphi(Q)$ is relatively compact and graph $\Gamma_{\varphi}$ is closed. Therefore, the mapping $\varphi$ is u.s.c., compact and, in particular, with compact values.

(iv) Finally, we will show that the mapping $\varphi$ has convex values.

Let $u_{1}, u_{2}$ be two distinct solutions of problem (15) associated with measurable selections $f_{1, q}, f_{2, q} \subset F_{q}$. Then, for all $t \in J$, we have

$$
\begin{aligned}
& u_{1}(t)=\int_{0}^{T} G_{1}(t, s) f_{1, q}(s) \mathrm{d} s \\
& u_{2}(t)=\int_{0}^{T} G_{1}(t, s) f_{2, q}(s) \mathrm{d} s .
\end{aligned}
$$

Let $\lambda \in[0,1]$ be arbitrary. Since $F$ is convex-valued, the same is true for $F_{q}$, and subsequently

$$
f_{q}(t)=\lambda f_{1, q}(t)+(1-\lambda) f_{2, q}(t)
$$

must be a measurable selection of $F_{q}$, i.e. $f_{q} \subset F_{q}$. We have

$$
\begin{aligned}
u(t) & =\lambda u_{1}(t)+(1-\lambda) u_{2}(t) \\
& =\lambda \int_{0}^{T} G_{1}(t, s) f_{1, q}(s) \mathrm{d} s+(1-\lambda) \int_{0}^{T} G_{1}(t, s) f_{2, q}(s) \mathrm{d} s \\
& =\int_{0}^{T} G_{1}(t, s)\left[\lambda f_{1, q}(s)+(1-\lambda) f_{2, q}(s)\right] \mathrm{d} s=\int_{0}^{T} G_{1}(t, s) f_{q}(s) \mathrm{d} s .
\end{aligned}
$$

Thus, $u(\cdot)$ must also be a solution of (15), by which the mapping $\varphi$ has convex values.

After all, applying Proposition 2, we obtain the existence of a fixed point of the multivalued mapping $\varphi$ which represents a solution of problem (13). However, because of the definitions of $F_{1}^{*}, F_{2}^{*}$, such a solution must be a solution of problem (12) as well as (9). 
Example 1 As an illustrative example of a Dirichlet problem satisfying rather implicit conditions (20)-(22), we can consider (9), where

$$
\begin{aligned}
& |a|=|b|=\frac{1}{4} \quad \Longrightarrow \quad k_{1}=\frac{1}{4}, \\
& x_{0}=x_{T}=\frac{1}{4} \quad \Longrightarrow \quad k_{2}=\frac{1}{4}, \quad k_{3}=0 \\
& T=2(\sqrt{2}-1) \quad \Longrightarrow \quad \frac{4}{T(T+4)}=1>\frac{1}{4}=k_{1} \quad(\text { see }(20)) .
\end{aligned}
$$

In this way, conditions (21), (22) take, for $D_{0}=\frac{1}{4}$, the form:

$$
\frac{\mathcal{P}}{2(\sqrt{2}-1)}+\left[M_{1}\left(\frac{1}{4}\right)+M_{2}\left(\frac{1}{4}\right)+|c|\right] \leq \frac{1}{8} .
$$

Taking, furthermore, $\mathcal{P} \leq \frac{\sqrt{2}-1}{16}$ and $|c|=\frac{1}{32}$, we get for $F_{1}(z):=f_{1} z^{m}$ and $F_{2}(z):=f_{2} z^{n}$ the inequality:

$$
\left|f_{1}\right|\left(\frac{1}{2}\right)^{m}+\left|f_{2}\right|\left(\frac{1}{2}\right)^{n} \leq \frac{1}{16}
$$

Hence, if $\left|f_{1}\right|,\left|f_{2}\right| \leq \frac{1}{16}$ and $m, n \geq 1$, then we arrive at (21), (22), because for $\left|f_{1}\right|=\left|f_{2}\right|=\frac{1}{16}$ and $m=n$, we have

$$
\left(\frac{1}{2}\right)^{m-1} \leq 1
$$

which holds for all $m \geq 1$.

After all, under these assumptions, problem (9) admits a solution $x(\cdot)$ such that

$$
\max _{t \in[0, T]}\left\{|x(t)|+\left|x^{\prime}(t)\right|\right\} \leq \frac{1}{2} .
$$

In fact, the same is true for (9), where $F_{1}, F_{2}$ are as above for $z \in\left[-\frac{1}{2}, \frac{1}{2}\right]$ and can be arbitrary outside of the interval $\left[-\frac{1}{2}, \frac{1}{2}\right]$.

Remark 1 If, additionally, a multivalued mapping $P$ is essentially bounded, then condition (20) can be replaced, for the same conclusion for (9), by

$$
\frac{8}{T(T+4)}>\max \{|a|,|b|\}
$$

and (21) with $\Delta_{1}\left(D_{0}\right)$ in (22) can be replaced by $\widetilde{D}_{0} \geq \Delta_{1}^{\prime}\left(\widetilde{D}_{0}\right)$, where

$$
\Delta_{1}^{\prime}\left(\widetilde{D}_{0}\right):=\frac{\left[\mathcal{P}_{1}+M_{1}\left(\widetilde{D}_{0}\right)+M_{2}\left(\widetilde{D}_{0}\right)+|a| k_{3}+|b| k_{2}+|c|\right] T(T+4)}{8-k_{1} T(T+4)},
$$

$\mathcal{P}_{1}:=\operatorname{ess}_{\sup }|P(t)|$, because

$$
\int_{0}^{T}\left|G_{1}(t, s)\right| \mathrm{d} s \leq \frac{T^{2}}{8} \quad \text { and } \quad \int_{0}^{T}\left|\frac{\partial G_{1}}{\partial t}(t, s)\right| \mathrm{d} s \leq \frac{T}{2} .
$$

Thus, the same conclusion holds for (9) with $D$ replaced by $\widetilde{D}=\widetilde{D}_{0}+k_{2}+k_{3}$. 
Remark 2 If, additionally, a multivalued mapping $F_{2}$ is bounded, i.e. $M_{2}:=\max _{z \in \mathbb{R}}\left|F_{2}(z)\right|$, and $a=0$, we can still improve the estimate for a solution of (9). Condition (20) can then be replaced by $\frac{4}{T^{2}}>|b|$, provided there still exists a positive constant $D_{1}$ such that

$$
D_{1} \geq \frac{T\left[\mathcal{P}+\left(M_{1}\left(D_{1}\right)+M_{2}+|b| k_{2}+|c|\right) T\right]}{4-T^{2}|b|} .
$$

Under these assumptions, problem (9) has a solution $x(\cdot)$ such that

$$
\begin{aligned}
& \max _{t \in J}|x(t)| \leq D_{1}+k_{2}, \\
& \max _{t \in J}\left|x^{\prime}(t)\right| \leq D_{2}+k_{3}:=\mathcal{P}+T\left[M_{1}\left(D_{1}\right)+M_{2}+|b|\left(D_{1}+k_{2}\right)+|c|\right]+k_{3},
\end{aligned}
$$

where $M_{1}\left(D_{1}\right):=\max _{|z| \leq D_{1}+k_{2}}\left|F_{1}(z)\right|, k_{2}:=\max \left\{\left|x_{0}\right|,\left|x_{T}\right|\right\}, k_{3}:=\frac{\left|x_{T}-x_{0}\right|}{T}$.

Similarly if, additionally, a multivalued mapping $F_{1}$ is bounded, i.e. $M_{1}:=\max _{z \in \mathbb{R}}\left|F_{1}(z)\right|$, and $b=0$, then condition (20) can be replaced by $\frac{1}{T}>|a|$, provided there still exists a positive constant $D_{2}$ such that

$$
D_{2} \geq \frac{\mathcal{P}+T\left(M_{1}+M_{2}\left(D_{2}\right)+|a| k_{3}+|c|\right)}{1-T|a|},
$$

where $M_{2}\left(D_{2}\right):=\max _{|z| \leq D_{2}+k_{3}}\left|F_{2}(z)\right|$. Here, problem (9) has a solution $x(\cdot)$ such that

$$
\begin{aligned}
& \max _{t \in J}|x(t)| \leq D_{1}+k_{2}:=\frac{T}{4}\left[\mathcal{P}+T\left(M_{1}+M_{2}\left(D_{2}\right)+|a|\left(D_{2}+k_{3}\right)+|c|\right)\right]+k_{2}, \\
& \max _{t \in J}\left|x^{\prime}(t)\right| \leq D_{2}+k_{3} .
\end{aligned}
$$

\section{Discussion of further possibilities}

Now, we will make comments on the existence of a solution of the problem (9), but this time considered in the following form:

$$
\left.\begin{array}{l}
x^{\prime \prime}(t)+a x^{\prime}(t)+b x(t) \in F\left(t, x(t), x^{\prime}(t)\right), \quad \text { for a.a. } t \in J, \\
x(0)=x_{0}, \quad x(T)=x_{T},
\end{array}\right\}
$$

where $F\left(t, x(t), x^{\prime}(t)\right):=P(t)+F_{1}(x(t))+F_{2}\left(x^{\prime}(t)\right)-c \operatorname{Sgn} x^{\prime}(t)$ is a u-Carathéodory multivalued mapping and $L x(t):=x^{\prime \prime}(t)+a x^{\prime}(t)+b x(t)$. This allows us to consider different Green's functions associated with the complete linear differential operator.

In this way, the related solvability criteria are as follows:

(1) For $a^{2}-4 b>0$ : $D_{0} \geq \Delta_{2}\left(D_{0}\right)$, where

$$
\begin{aligned}
& \begin{aligned}
\Delta_{2}\left(D_{0}\right):= & \frac{e^{\left(\lambda_{1}-\lambda_{2}\right) T}}{\sqrt{a^{2}-4 b}}\left[1+\left|\lambda_{1}\right|+\left|\lambda_{2}\right|\right] \\
& \times\left[\mathcal{P}+T\left(M_{1}\left(D_{0}\right)+M_{2}\left(D_{0}\right)+|c|+|a| k_{3}+|b| k_{2}\right)\right], \\
\lambda_{1}=\frac{-a+\sqrt{a^{2}-4 b}}{2}, & \lambda_{2}=\frac{-a-\sqrt{a^{2}-4 b}}{2}
\end{aligned} \\
& \text { (2) For } a^{2}-4 b=0:
\end{aligned}
$$

$$
\begin{aligned}
\Delta_{3}\left(D_{0}\right):= & e^{\frac{|a|}{2} T}\left[1+\frac{T}{4}(1+2|a|)\right] \\
& \times\left[\mathcal{P}+T\left(M_{1}\left(D_{0}\right)+M_{2}\left(D_{0}\right)+|c|+|a| k_{3}+|b| k_{2}\right)\right] .
\end{aligned}
$$


(3) For $a^{2}-4 b<0$ and $T \neq \frac{2 \pi k}{\sqrt{4 b-a^{2}}}, k \in \mathbb{N}: D_{0} \geq \Delta_{4}\left(D_{0}\right)$, where

$$
\begin{aligned}
\Delta_{4}(D):= & \frac{e^{\frac{|a|}{2} T}\left[2+|a|+\sqrt{4 b-a^{2}}\right]}{\sqrt{4 b-a^{2}}\left|\sin \left(\frac{T}{2} \sqrt{4 b-a^{2}}\right)\right|} \\
& \times\left[\mathcal{P}+T\left(M_{1}\left(D_{0}\right)+M_{2}\left(D_{0}\right)+|c|+|a| k_{3}+|b| k_{2}\right)\right] .
\end{aligned}
$$

In all the cases (1), (2), (3), the problem (9) admits a solution $x(\cdot)$ such that $\max _{t \in J}\{|x(t)|+$ $\left.\left|x^{\prime}(t)\right|\right\} \leq D_{0}+k_{2}+k_{3}$, where $k_{2}:=\max \left\{\left|x_{0}\right|,\left|x_{T}\right|\right\}, k_{3}:=\frac{\left|x_{T}-x_{0}\right|}{T}$.

In view of Remark 2 and the estimates above, we can immediately formulate the following corollary for a Lebesgue integrable selection $p \subset P$ and $F_{1}(x):=d \sin x$.

Corollary 1 Let $b, c, d, x_{0}, x_{T}$ and $T>0$ be real constants, and $p:[0, T] \rightarrow \mathbb{R}$ be a Lebesgue integrable mapping, where $\mathcal{P}:=\int_{0}^{T}|p(t)| \mathrm{d} t<\infty$. Then there exists a Filippov solution $x(\cdot)$ of problem

$$
\left.\begin{array}{l}
x^{\prime \prime}(t)+b x(t)+c \operatorname{sgn} x^{\prime}(t)+d \sin x(t)=p(t), \quad \text { for almost all } t \in[0, T], \\
x(0)=x_{0}, \quad x(T)=x_{T},
\end{array}\right\}
$$

such that, for $0<b \neq\left(\frac{k \pi}{T}\right)^{2}, k \in \mathbb{N}$,

$$
\begin{aligned}
& \max _{t \in[0, T]}|x(t)| \leq \frac{\mathcal{P}+T\left[|b| k_{2}+|c|+|d|\right]}{\sqrt{b}|\sin (\sqrt{b} T)|}+k_{2}, \\
& \max _{t \in[0, T]}\left|x^{\prime}(t)\right| \leq \frac{\mathcal{P}+T\left[|b| k_{2}+|c|+|d|\right]}{|\sin (\sqrt{b} T)|}+\frac{\left|x_{T}-x_{0}\right|}{T} .
\end{aligned}
$$

For $b<0$ and $b=0$, we obtain the respective estimates

$$
\begin{aligned}
& \max _{t \in[0, T]}|x(t)| \leq \frac{e^{2 \sqrt{-b} T}}{2 \sqrt{-b}}\left[\mathcal{P}+T\left(|b| k_{2}+|c|+|d|\right)\right]+k_{2}, \\
& \max _{t \in[0, T]}\left|x^{\prime}(t)\right| \leq e^{2 \sqrt{-b} T}\left[\mathcal{P}+T\left(|b| k_{2}+|c|+|d|\right)\right]+\frac{\left|x_{T}-x_{0}\right|}{T}
\end{aligned}
$$

and

$$
\begin{aligned}
& \max _{t \in[0, T]}|x(t)| \leq \frac{T[\mathcal{P}+T(|c|+|d|)]}{4}+k_{2}, \\
& \max _{t \in[0, T]}\left|x^{\prime}(t)\right| \leq \mathcal{P}+T(|c|+|d|)+\frac{\left|x_{T}-x_{0}\right|}{T},
\end{aligned}
$$

where $k_{2}=\max \left\{\left|x_{0}\right|,\left|x_{T}\right|\right\}$.

Remark 3 Observe that, for $b=0$, the conclusion of Corollary 1 reduces to the Dirichlet problem for a forced pendulum with a dry friction while, for $d=0$, the result concerns the Dirichlet problem for a forced 'linear' oscillator with a dry friction. Moreover, the nonresonance condition $b \neq\left(\frac{k \pi}{T}\right)^{2}, k \in \mathbb{N}$, distinguished in Corollary 1 in three cases $(b>0$, $b<0, b=0)$, is evidently optimal, when comparing it with all its analogies discussed above (cf. (20), Remark 1 and Remark 2). 
Finally, we can also consider a particular case with $c=0$ of the Dirichlet problem (9) in a vector form in $\mathbb{R}^{n}$, i.e. let $a, b(c=0)$ and $T>0$ remain real constants, but $x_{0}, x_{T} \in \mathbb{R}^{n}$, $F_{1}: \mathbb{R}^{n} \multimap \mathbb{R}^{n}, F_{2}: \mathbb{R}^{n} \multimap \mathbb{R}^{n}$ be u.s.c. with convex and compact values and $P: J \multimap \mathbb{R}^{n}$ be an Aumann (component-wise)-integrable mapping. In this way, dry friction terms can be involved, after their Filippov's regularization, in some components of $F_{2}$.

All the above statements can then be reformulated for the vector case in the appropriate norm $\|\cdot\|$, when the truncated vector analogies of $F_{1}^{*}, F_{2}^{*}$ in (13) are defined as follows $(i=1,2)$ :

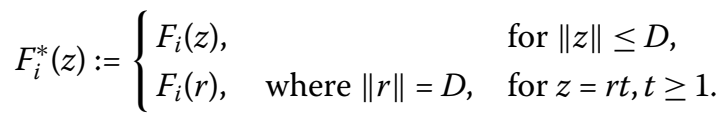

Observe that in this way $F_{i}^{*}$ is obviously u.s.c. with nonempty, compact, convex values, provided $\left.F_{i}\right|_{\bar{B}_{D}}$ is so, where $\bar{B}_{D}:=\left\{z \in \mathbb{R}^{n} \mid\|z\| \leq D\right\}$. Moreover, $M_{i}(D):=\max _{z \in \bar{B}_{D}}\left\|F_{i}(z)\right\|=$ $\max _{z \in \mathbb{R}^{n}}\left\|F_{i}^{*}(z)\right\|, i=1,2$.

Since $\bar{B}_{D}$ is an absolute retract, there exists a continuous retraction $r: \mathbb{R}^{n} \rightarrow \bar{B}_{D}$ such that $\left.r\right|_{\bar{B}_{D}}=\left.\mathrm{id}\right|_{\bar{B}_{D}}$ (see e.g. Chapter I.2 in [14]). Therefore, we can alternatively replace $F_{i}^{*}(z)$ by $F_{i}(r(z))$, where $(i=1,2)$

$$
F_{i}(r(z)):= \begin{cases}F_{i}(z), & \text { for }\|z\| \leq D \\ F_{i}(r(z)), & \text { for }\|z\| \geq D\end{cases}
$$

because the compositions $F_{i}(r(z)), i=1,2$, must also be u.s.c. maps with convex, compact values with the same properties (see e.g. Chapter I.3.3 in [14]).

\section{Competing interests}

The authors declare that they have no competing interests.

\section{Authors' contributions}

All authors contributed equally in this article. They read and approved the final manuscript.

\section{Acknowledgements}

The first author was supported by the grant No. 14-06958S 'Singularities and impulses in boundary value problems for nonlinear ordinary differential equations' of the Grant Agency of the Czech Republic. The second author was supported by the grant IGA Mathematical Models, IGA_PrF_2014028 and by the grant No. 14-06958S 'Singularities and impulses in boundary value problems for nonlinear ordinary differential equations' of the Grant Agency of the Czech Republic.

\section{Endnote}

a Because of the terms $c \operatorname{sgn} x^{\prime}(t)$ resp. $c \operatorname{Sgn} x^{\prime}(t)$, the related equations resp. inclusions are no longer linear but, for the sake of simplicity, we reserve for them this name.

Received: 10 April 2015 Accepted: 8 June 2015 Published online: 24 June 2015

\section{References}

1. Mawhin, J: Problèmes de Dirichlet variationnels non linéaires. Partie 1 des comptes rendus du cours d' été OTAN 'Variational Methods in Nonlinear Problems'. Presses University Montreal, Montreal (1987)

2. Andres, J, Sanchez, L: A note on vector boundary value problems. Nonlinear Differ. Equ., TMA 3(1-2), 49-58 (1997)

3. Bernfeld, SR, Lakshmikantham, V: An Introduction to Nonlinear Boundary Value Problems. Academic Press, New York (1974)

4. Erbe, LH, Knobloch, HW: Boundary value problems for systems of second order differential equations. Proc. R. Soc. Edinb. A 101(1-2), 61-76 (1985)

5. Fabry, C, Habets, P: The Picard boundary value problem for nonlinear second order vector differential equations. J. Differ. Equ. 42(2), 186-198 (1981)

6. Granas, A, Guenther, RB, Lee, JW: Nonlinear Boundary Value Problems for Ordinary Differential Equations. Dissertationes Math., vol. 244. PWN, Warsaw (1985)

7. Habets, P, Schmitt, K: Nonlinear boundary value problems for system of differential equations. Arch. Math. (Basel) 40(1), 441-446 (1983) 
8. Hartman, P: On boundary value problems for systems of ordinary nonlinear second order differential equations. Trans. Am. Math. Soc. 96(3), 493-509 (1960)

9. Knobloch, HW, Schmitt, K: Nonlinear boundary value problems for systems of differential equations. Proc. R. Soc. Edinb. A 78(1-2), 139-159 (1977)

10. Mawhin, J: Boundary value problems for nonlinear second-order vector differential equations. J. Differ. Equ. 16(2), 257-269 (1974)

11. O'Regan, D: Boundary value problems for second and higher order differential equations. Proc. Am. Math. Soc. 113(3), 761-775 (1991)

12. Scorza-Dragoni, G: Sul problema dei valori ai limiti per i sistemi di equazioni differenziali del secondo ordine. Boll. Unione Mat. Ital. 14, 225-230 (1935)

13. Scorza-Dragoni, G: Sui sistemi di equazioni integrali non lineari. Rend. Semin. Mat. Univ. Padova 7, 1-35 (1936)

14. Andres, J, Górniewicz, L: Topological Fixed Point Principles for Boundary Value Problems. Kluwer Academic, Dordrecht (2003)

15. Filippov, AF: Differential Equations with Discontinuous Righthand Sides. Kluwer Academic, Dordrecht (1988)

16. Kunze, M: Non-Smooth Dynamical Systems. Lecture Notes in Mathematics, vol. 1744. Springer, Berlin (2000)

17. Monteiro Marques, MDP: Differential Inclusions in Nonsmooth Mechanical Problems: Shocks and Dry Friction. Birkhäuser, Basel (1993)

18. Popp, K, Hinrichs, N, Oestreich, M: Dynamical behaviour of a friction oscillator with simultaneous and external excitation. Sādhanā 20(2-4), 627-654 (1995)

19. Stewart, DE: Rigid-body dynamics with friction and impact. SIAM Rev. 42(1), 3-39 (2000)

20. Zhuravlev, VP: On the history of the dry friction law. Mech. Solids 48(4), 364-369 (2013)

21. Dugundji, J, Granas, A: Fixed Point Theory. Springer, Berlin (2003)

22. Lasota, A, Opial, Z: An application of the Kakutani-Ky Fan theorem in the theory of ordinary differential equations. Bull. Acad. Pol. Sci., Sér. Sci. Math. Astron. Phys. 13(11-12), 781-786 (1965)

23. Andres, J, Malaguti, L, Pavlačková, M: Hartman-type conditions for multivalued Dirichlet problem in abstract spaces. Discrete Contin. Dyn. Syst., Ser. A (to appear)

24. Cubiotti, P, Yao, JC: Two-point problem for vector differential inclusions with discontinuous right-hand side. Appl. Anal. 93(9), 1811-1823 (2014)

25. Granas, A, Guenther, RB, Lee, JW: Some general existence principles in the Carathéodory theory of nonlinear differential systems. J. Math. Pures Appl. 70(2), 153-196 (1991)

26. Kožušníková, M: A bounding functions approach to multivalued Dirichlet problem. Atti Semin. Mat. Fis. Univ. Modena Reggio Emilia 55, 1-19 (2007)

27. Lee, JW, O'Regan, D: Existence of solutions to some initial value, two point and multipoint boundary value problems with discontinuous nonlinearities. Appl. Anal. 33(1-2), 57-77 (1989)

28. Nistri, P: Positive solutions of non-linear eigenvalue problem with discontinuous non-linearity. Proc. R. Soc. Edinb. A 83(1-2), 133-145 (1979)

29. Pavlačková, M: A bound sets technique for Dirichlet problem with an upper-Carathéodory right-hand side. Acta Univ Palacki. Olomuc., Fac. Rerum Nat., Math. 49(2), 95-106 (2010)

30. Pavlačková, M: A Scorza-Dragoni approach to Dirichlet problem with an upper-Carathéodory right-hand side. Topol. Methods Nonlinear Anal. 44(1), 239-247 (2014)

31. Pruszko, T: Some Applications of the Topological Degree Theory to Multi-Valued Boundary Value Problems. Dissertationes Math., vol. 229. PWN, Warsaw (1984)

32. Stuart, CA: Differential equations with discontinuous non-linearities. Arch. Ration. Mech. Anal. 63(1), 59-75 (1976)

33. Šenkyřík, M, Guenther, RB: Boundary value problems with discontinuities in the spatial variable. J. Math. Anal. Appl. 193(1), 296-305 (1995)

34. Zuev, AV: On the Dirichlet problem for a second-order ordinary differential equation with discontinuous right-hand side. Differ. Equ. 42(3), 340-346 (2006)

35. Castaing, C, Valadier, M: Convex Analysis and Measurable Multifunctions. Springer, Berlin (1977)

36. Appell, J, De Pascale, E, Nguyen, HT, Zabreiko, PP: Multi-Valued Superpositions. Dissertationes Math., vol. 345. PWN, Warsaw (1995)

37. Aumann, RJ: Integrals of set-valued functions. J. Math. Anal. Appl. 12(1), 1-12 (1965)

38. Stakgold, I: Green's Functions and Boundary Value Problems. Wiley, New York (1979)

39. Aubin, J-P, Cellina, A: Differential Inclusions. Springer, Berlin (1984)

40. Vrabie, II: Compactness Methods for Nonlinear Evolutions. Longman, Harlow (1990) 